Rabaska

Revue d'ethnologie de l'Amérique française

\title{
Le Romancero du Canada : une synthèse à la croisée des chemins
}

\section{Jean-Pierre Pichette}

Volume 13, 2015

Présence de Marius Barbeau : l'invention du terrain en Amérique

française. Autour d’un legs centenaire (1914-2014)

URI : https://id.erudit.org/iderudit/1033751ar

DOI : https://doi.org/10.7202/1033751ar

Aller au sommaire du numéro

Éditeur(s)

Société québécoise d'ethnologie

ISSN

1703-7433 (imprimé)

1916-7350 (numérique)

Découvrir la revue

Citer cet article

Pichette, J.-P. (2015). Le Romancero du Canada : une synthèse à la croisée des chemins. Rabaska, 13, 77-88. https://doi.org/10.7202/1033751ar d'utilisation que vous pouvez consulter en ligne.

https://apropos.erudit.org/fr/usagers/politique-dutilisation/ 


\section{Barbeau et l'oralité : la chanson}

\section{Le Romancero du Canada : une synthèse à la croisée des chemins}

Jean-Pierre Pichette

Université Sainte-Anne, Pointe-de-l’Église et Société québécoise d'ethnologie

\section{Le livre}

En 1937, Marius Barbeau est un anthropologue bien établi au Musée national du Canada et il jouit d'une réputation enviable quand il publie aux éditions Beauchemin de Montréal et chez Macmillan à Toronto un livre important : le Romancero du Canada ${ }^{1}$. Quelques critiques lui reprochèrent son titre ésotérique, le substantif étranger romancero faisant difficulté. D'origine espagnole et passé en français vers 1831, le mot aura fini par signifier un recueil de chansons de tradition orale, ainsi que le confirme l'édition en 1904 du Romancéro populaire de la France, dont le sous-titre Choix de chansons populaires françaises éclaire l'interprétation. C'est donc au répertoire des chansons traditionnelles du Canada français que s'attache ici l'auteur².

\section{Disposition}

L'entrée en matière du recueil est concise, réduite à l'essentiel. Le lecteur y apprend que les chansons retenues « sont des échantillons » de la grande collection d'" environ sept mille textes et plus de quatre mille mélodies recueillies pour la plupart au phonographe » que l'auteur a réunie pour le compte du Musée national du Canada et, puisqu'il destine son livre « au grand public et aux artistes, et le nombre de ses pages étant restreint, les

1. Marius Barbeau, Romancero du Canada, [Montréal], Éditions Beauchemin ; [Toronto, Macmillan], 1937, $254 \mathrm{p}$.

2. À la décharge de Barbeau, il faut remarquer que des titres plus familiers, comme « Chansons populaires du Canada », «Chants populaires du Canada » ou « Chansons populaires du vieux Québec », n'étaient plus disponibles depuis que Gagnon, Massicotte et Barbeau lui-même les avaient employés. 
variantes - souvent intéressantes - des textes et des mélodies n'ont pu être transcrites ${ }^{3}$ ». Barbeau laisse alors la place à la préface datée de juin 1936, à Paris, d'une ethnomusicologue française de grande réputation, Marguerite Béclard d'Harcourt ; celle-ci apprécie la très grande valeur de la tradition canadienne-française et note la persistance de modes et de rythmes variés qui ont échappé aux contraintes de l'écriture musicale.

Chaque chapitre s'ordonne selon un modèle précis. Sous le titre de la chanson, apparaît d'abord le relevé musical de la pièce à l'étude. D'après l'auteur, les mélodies présentées « n'ont subi de légères retouches que dans le cas où leur déformation venait d'une gaucherie des chanteurs ».

À la suite de la mélodie, Barbeau dispose les paroles de toute la chanson. Contrairement aux mélodies, il adopte ici une voie différente : « Les paroles ont été reconstituées en un texte critique, d'après les diverses versions canadiennes enregistrées ». Des commentaires précisent alors, en des rédactions plus ou moins élaborées de deux à huit pages, la nature, le genre et l'usage de la chanson, sa versification, exposent son ancienneté et son origine française, et justifient sa présence au Canada ; ils décrivent aussi la « formule rythmique » et s'achèvent par une « analyse musicale » préparée par Marguerite Béclard d'Harcourt. En appendice, un « catalogue des versions » énumère toutes les variantes canadiennes connues, jusqu'à vingt-deux dans un cas, suivant une géographie d'ouest en est, incluant quelquefois l'Alberta, le Manitoba et l'Ontario, mais balayant surtout le Québec (de la Gatineau à la Gaspésie, rive nord puis rive sud) et se déployant même à l'occasion jusqu'à l'Île-du-Prince-Édouard ; chaque version numérotée et localisée est identifiée par le patronyme du collecteur et datée ; puis le nom de l'informateur apparaît avec la source de son information, si elle est connue, son numéro d'enregistrement phonographique et son titre. Quand les circonstances l'exigent, d'autres détails font l'objet d'annotations sobres sur les particularités de la chanson, son refrain, ses couplets et sa mélodie. Le « catalogue » consigne en outre les versions européennes, en majorité françaises, et, parce qu'elles sont d'ordinaire publiées, dans l'ordre chronologique de leur parution; les annotations tiennent alors compte des textes eux-mêmes, car, en raison de leur méthodologie obsolète, les données des références européennes sont moins abondantes et les sources indéfinies.

\section{Ordonnance thématique}

Bien que l'anthologie n'affiche aucune division expresse, les analyses de

3. Marius Barbeau, Romancero du Canada, « Avant-propos », p. [5]. Dans une entrevue qu'il donna en 1965, Barbeau dira : « Je me suis efforcé d'inclure les meilleurs exemples du répertoire canadien, en me basant sur ce qui se trouvait en ma possession »; voir Marius Barbeau, Je suis un pionnier, dans Oracle, Ottawa, Musée national de l'Homme, nº 43 1982, p. [7]. 
Marius Barbeau révèlent néanmoins le principe d'ordre tacite, qui organise toute cette matière et la regroupe implicitement en sept séries thématiques. La première série se compose de chansons de voyageurs ou de canotiers, parfois à sujet historique ou véritablement marin, destinées à rythmer les efforts des rameurs. Une deuxième série réunit des complaintes : les amours malheureuses de l'amant inconsolable du décès de sa belle ou de la fille mariée contre son gré à un riche marchand précèdent les tragédies les plus meurtrières : le suicide de l'accouchée à qui on a caché le trépas de son mari, la décapitation de la fiancée infidèle, etc. Les séparations d'amants forment la troisième série alors que la quatrième rassemble des chansons de canotiers et de métiers qui ont l'amour pour thème principal. Les cantiques populaires se rangent dans une série particulière, la cinquième : ces ballades racontent le miracle de saint Nicolas ressuscitant un enfant, la parabole évangélique du mauvais riche ou la passion de Jésus-Christ, la quête légendaire de la sainte Vierge et le martyre de sainte Catherine. Une chanson de voyageurs, qui dit l'ennui d'un coureur de bois éloigné de sa belle, amorce, sur le mode mélancolique, la sixième série consacrée aux chansons de rossignols. La septième et dernière série associe des chansons destinées à la danse et dont la plupart ont un lien avec le mariage : proposition d'union demeurée sans réponse ou refusée au couturier, lamentation d'une maumariée jugeant que c'est folie de s'engager à un entêté ou misère d'un mari ivrogne battu par sa femme.

\section{Commentaires}

À propos de la chanson à l'étude, le commentateur en qualifie d'abord la nature par une précision destinée à mettre en évidence tantôt ses utilisateurs - « chanson de voyageurs » ou « de canotiers », « chanson de fileuses », « ronde », « chanson de danse », « chanson de quête », « chanson à boire »-, tantôt son scénario ou son genre - « complainte », « chanson lyrique », « chanson d'amour », " aubade », « pastourelle », « chanson de mariage », « chanson de maumariée », " cantique populaire ». Il ajoutera à l'occasion une appréciation d'ordre esthétique.

Il donne ensuite des indications sur sa vitalité au Canada - dénombrement des versions, haute fréquence ou rareté de nos jours -, sur sa répartition géographique : "Cette jolie pastourelle de France, dit-il de Je te ferai demoiselle, semble ne s'être conservée qu'au centre de Québec »; par contre, M'amie que j'aime tant a été recueillie « dans toutes les parties du Canada français, depuis Beauharnois jusqu'à la Baie-de-Chaleur ». Puis il s'intéresse à la versification, au scénario et aux variantes des textes poétiques tout autant qu'il discute des variations mélodiques et de leurs qualités esthétiques. Il constate aussi l'origine, rarement locale, ordinairement française de la chanson 
et, selon les régions où les versions ont été moissonnées, propose à l'occasion une explication de sa présence à Québec, à Montréal ou en Acadie, par la souche provinciale du peuplement français.

S'il a eu la chance d'en lire des variantes dans les recueils français, il se lance alors dans la comparaison entre les versions des deux continents et tente souvent d'en apprécier l'ancienneté. Il sonde l'histoire, résume, commente et critique certains détails à la lumière des versions canadiennes qu'il a dénichées ; celles-ci lui permettent parfois de contester des datations jugées trop récentes et de les repousser d'un siècle ou deux. Pour ce faire, il dispose d'une riche bibliographie et il réfère ainsi son lecteur à environ cinquantecinq auteurs, parmi les meilleurs spécialistes de la chanson française d'alors, et cite en outre des textes parallèles suisses, italiens, espagnols, portugais, allemands et anglais. Sa recherche documentaire paraît vraiment admirable à une époque où les bibliothèques canadiennes et les moyens de communication étaient plutôt rudimentaires ; par comparaison, sa connaissance des travaux franco-européens sur la chanson folklorique dépasse de loin celle qu'eurent, de la tradition franco-canadienne, les spécialistes français qui l'ont suivi.

Bien sûr, Barbeau pressentait que, pour l'immense majorité, ces chansons de tradition orale étaient venues au Canada par l'émigration française. Mais avait-il prévu que certains airs français recueillis en Amérique ne se retrouveraient plus en France ? Que dix-sept des cinquante chansons de son recueil, soit plus du tiers, n'y seraient pas attestées ? Et que, parmi les trente-deux autres, dix-huit présenteraient un nombre de versions inférieur à celui du Canada, une douzaine de morceaux affichant plus d'attestations françaises ? Ces faits, qu'enregistre avec grand intérêt l'auteur ébahi, prennent bientôt l'allure d'un complet renversement : il appert que ce petit répertoire, constitué pour l'essentiel sous sa direction entre 1916 et 1931, se ramifie en 391 versions canadiennes contre seulement 244 pour la France et les pays d'Europe ; plus encore, à lui seul, Marius Barbeau aurait réuni plus de documents au Canada (260 versions), soit les deux tiers de l'inventaire canadien présenté, qu'il n'en a trouvé dans toute l'Europe.

\section{Une tradition intacte}

Ces statistiques ont étayé certaines des observations qui confortent l'auteur sur l'importance et l'originalité de son entreprise. Des remarques, toutes simples en apparence, comme « Cette allègre chanson de danse n'est connue qu'en une seule version » ou « Elle nous vient sans doute de France - dont les recueils, cependant, ne semblent pas la contenir », font ailleurs place à l'étonnement : « elle n'est plus connue en France, d'où elle nous est pourtant venue, avec les anciens colons » ou « Chose curieuse, cette jolie composition semble à 
peine avoir survécu dans le répertoire de France ». En y regardant de près, Barbeau notait, dès les premières pages, des lacunes dans la transmission de la chanson traditionnelle en France même.

Disséminés à une quinzaine d'endroits dans le livre, les commentaires de cette nature révèlent à quel point ce phénomène de l'érosion de la chanson traditionnelle en France avait bouleversé Barbeau qui s'en préoccupait constamment et ne pouvait s'expliquer autrement sa rareté dans les recueils européens. Voulant déborder la curiosité, il passe alors à la comparaison des traditions communes et il ne tarde pas à pointer la qualité supérieure des versions canadiennes : « Cette belle complainte a mieux survécu chez nous qu'en France ». Pourtant, l'origine française ne peut être remise en doute, mais c'est lorsque la tradition canadienne se montre vigoureuse que la lacune française se fait plus criante. Pour expliquer ce phénomène, Barbeau s'appuie sur le tempérament propre du Canadien et sur sa situation géographique : «Québec et l'Acadie sont restées jusqu'aujourd'hui des provinces purement françaises, en tant que conservatrices de la tradition. Ce qui souvent s'est perdu là-bas s'est conservé ici, grâce à l'isolement et au conservatisme inné du colon canadien ». Ces gloses de Barbeau correspondent tout à fait aux remarques enthousiastes et sans ambages de sa préfacière, Marguerite Béclard d'Harcourt, qui les autorise, les sanctionne à l'avance et même les renforce.

\section{Étapes de la recherche}

En plus des remarques sur les chansons elles-mêmes et de son discours sur l'état supérieur de leur conservation au Canada, Barbeau ajoute des notes plus circonstancielles qui ont un intérêt certain, mais de tout autre ordre. Il s'agit sans doute aussi d'équilibrer les commentaires, surtout là où le petit nombre de versions canadiennes et l'absence de versions européennes rendraient la comparaison impossible. L'auteur met ainsi en valeur les recherches de ses prédécesseurs au Canada, rappelle ses propres enquêtes, présente son réseau de collaborateurs, évoque ses rencontres avec ses informateurs et décrit ses efforts de diffusion de la chanson populaire. De ce point de vue et sans en avoir l'air, le Romancero du Canada se mue en véritable traité de l'histoire des recherches sur la chanson populaire au Canada français.

\section{Les pionniers}

À l'occasion, Marius Barbeau signale les premiers témoignages connus sur la chanson populaire, il sollicite les chansonniers publiés et reconnaît l'antériorité d'Hubert Larue, l'auteur de la première étude en ce domaine. À l'égard des Chansons populaires du Canada d'Ernest Gagnon, ses sentiments sont ambivalents. S'il loue d'une part l'élargissement du « contact entre le 
terroir et le village » que marquait ce recueil en 1865, il déplore toutefois son impact négatif pour la recherche, car « le filon rural de nos traditions semblait avoir été épuisé d'un seul trait », et on " s'arrêta là tout court, bien à tort ». Par la suite, « on n'a guère popularisé chez nous, par le livre et le journal, que les chansons déjà recueillies et publiées, comme $\dot{A}$ la claire fontaine, $\dot{A}$ Saint-Malo, et une douzaine d'autres ", sans poursuivre la cueillette. L'ambition de « renouveler » le chansonnier de Gagnon aiguilla Barbeau vers un tout autre répertoire, ce qui explique que le Romancero du Canada ne contienne que trois références aux chansons publiées par ce devancier. En outre, il ne citera plus ces pionniers que pour noter les limites de leur répertoire. Loin d'être désobligeantes, ses remarques justifiaient la nécessité de l'entreprise qu'il avait lancée et la valeur des collections qu'il déposa au Musée national du Canada. Fruit d'une intuition fondamentale, qui s'éveilla en 1912 et que ses recherches intensives sont venues confirmer, la redécouverte de la chanson que Marius Barbeau a opérée, mais d'une chanson vivante en marge des élites, allait vraiment raviver la connaissance de ce patrimoine oral en le révélant de l'intérieur, du point de vue des témoins, par des enquêtes de terrain menées à sa source même.

\section{Les interprètes}

Ayant reconnu les mérites et les limites de ses devanciers, l'auteur entend livrer à petites doses, en coulant des notes discrètes parmi ses commentaires, les souvenirs des informateurs qui lui ont communiqué les chansons de son immense collection. Par des anecdotes choisies, tirées de son expérience et de celle de Massicotte, Barbeau brosse le portrait d'une quinzaine d'entre eux et évoque les rencontres qui ont favorablement marqué le projet qu'il portait. L'image de son père, Charles Barbeau, lui revient dès les premières pages à propos de La Courte Paille comme aussi de Toi qui chantes le jour et la nuit, « une des premières chansons que j'aie entendu chanter dans mon enfance ».

Sa mémoire le ramène bientôt à l'année 1916, celle où Charlevoix lui révéla, dans son habitat et son équipage traditionnels, la véritable chanson de tradition orale. C'est là que le vieil Édouard Hovington lui communiqua en une semaine quatre-vingt-dix chansons de voyageur, confondant ceux qui prétendaient que « ces chansons du Nord-Ouest [étaient] maintenant perdues »; que l'aveugle Louis Simard, dont la prestation publique lui suggéra les soirées qu'il organisera plus tard, lui en chanta autant en deux jours; et que $\mathrm{M}^{\mathrm{me}}$ Jean « Français » Bouchard, « la meilleure diseuse de complaintes du comté de Charlevoix », gagnera son admiration. De son exploration du Bas-Saint-Laurent, en 1918, il retient quelques surprises : tel ce Gilbert " Marin » Dumas qui interpréta Lisette d'une manière "si émouvante, si archaïque » qu'il crut « à un jongleur tombé des pages enluminées de 
quelque parchemin moyenâgeux » ; ou ce François Saint-Laurent, qui défila trois cent dix chansons méthodiquement rangées dans sa mémoire selon les points cardinaux ; et Alcide Léveillé, qui lui en communiqua une centaine. Il convoque encore trois femmes, chacune porteuse de deux cents chansons, que des terrains additionnels en Gaspésie et à l'île d'Orléans lui firent connaître en 1923 et en 1925 : M $^{\text {me }}$ Zéphirin Dorion, une Acadienne dans la quarantaine, qui lui chanta une version unique connue d'elle seule ; $\mathrm{M}^{\mathrm{me}}$ Pétronille Deblois-Dompierre, une collectionneuse nonagénaire qui avait compilé quatre chansonniers manuscrits ; et $\mathrm{M}^{\text {me }}$ Jean-Baptiste Leblond, « la meilleure chanteuse de l'île d'Orléans », qui lui confia une version isolée du Martyre de sainte Catherine.

Dans ce panorama, Barbeau fait aussi place aux quatre meilleures sources que son collaborateur É.-Z. Massicotte avait enregistrées à Montréal : Charles Belleau, de Québec, et Joseph Rousselle, de Saint-Denis de Kamouraska, qui connaissait cent cinquante chansons, Vincent-Ferrier de Repentigny, de Saint-Timothée de Beauharnois, véritable phénomène qui savait plus de trois cents chansons, et Philéas Bédard, de Saint-Rémi de Napierville, un artiste populaire que Barbeau interrogea aussi.

\section{Les collaborateurs}

Que Barbeau ait rouvert ce vaste pan de l'oralité populaire ne fait aucun doute. Mais, très tôt, il a su intéresser à son projet une réelle équipe de collaborateurs, qui prolongeaient sa quête dans les régions du Canada français. Le premier et le plus constant fut Édouard-Zotique Massicotte (1867-1947). Cet archiviste de Montréal s'intéressait à la chanson depuis 1883 sans avoir pu trouver de débouché à ses travaux. Son association à Barbeau en 1917 lui procurait enfin le stimulant essentiel. Dès la première rencontre, au cours de laquelle il chanta quelques chansons dont L'Hirondelle, messagère des amours, il s'enthousiasma au point que, deux ans plus tard, les deux compères publiaient le fruit de leur collaboration : Chants populaires du Canada (Première série). Recueillis par É.-Z. Massicotte et préparés par C.-Marius Barbeau, formant un numéro entier du Journal of American Folk-Lore ${ }^{4}$. Après une douzaine d'années d'échanges assidus (1917-1928), Massicotte avait accumulé 2292 textes de chansons dont 1521 mélodies au phonographe 5 . Barbeau évoque brièvement la figure du père Archange Godbout, o.f.m., qui récolta environ deux cents chansons du centre du Québec entre 1917 et 1919. La coopération avec Adélard Lambert (1867-1946), de Yamachiche,

4. Chants populaires du Canada (Première série). Recueillis par É.-Z. Massicotte et préparés par C.-Marius Barbeau, dans The Journal of American Folk-Lore, vol. 32, nº 123, January-March 1919, 89-i p.

5. Luc Lacourcière, «É.-Z. Massicotte. Son œuvre folklorique, Les Archives de folklore, vol. 3, [Montréal], Éditions Fides, 1948, p. 7-12. 
fut durable. Revenu s'établir au Canada après l'émigration de sa famille aux États-Unis, ce « collaborateur industrieux » transcrivit de mémoire, entre 1919 et 1928 , les contes et chansons qu'il avait entendus de sa mère durant son séjour étatsunien. Enfin, Barbeau rapporte sa brève association avec un correspondant acadien, l'abbé Pierre-Paul Arsenault (1866-1927) de l'Île-duPrince-Édouard, avec qui il entra en relation en 1924. Ce curé avait consigné la première collection acadienne.

\section{La promotion de la chanson}

Non seulement Barbeau fut-il un rassembleur de ces trésors du patrimoine oral, mais encore s'en fit-il le promoteur le plus ardent en toute circonstance. En commentant les pièces de son Romancero, il ne perd aucune occasion de signaler les œuvres nouvelles qu'elles ont inspirées et les exécutions qu'on en a faites. Barbeau fit encore davantage. Avec la complicité d’É.-Z. Massicotte, il organisa la première séance publique de folklore à Montréal en mars 1919, inspirée par l'exemple du chanteur Louis Simard; il en résultera une autre publication: Veillées du bon vieux temps à la Bibliothèque SaintSulpice. L'originalité de ces concerts résidait dans la prestation d'interprètes populaires et non d'artistes professionnels. La répétition de ces soirées dans d'autres villes du Québec d'alors fera naître les trois éditions du Festival de la chanson et des métiers du terroir (mai 1927-1928), modifié en Festival de la chanson, des danses et des métiers du terroir (octobre 1930), qui se tiendront au Château Frontenac de Québec, en association avec la compagnie de chemin de fer du Pacifique canadien. Durant ces trois saisons, il réunira pour le bénéfice des touristes de passage à Québec, avec des artistes et des artisans populaires, des chanteurs et musiciens professionnels.

Pour Barbeau - et en cela, il confirme la destination de son Romancero « au grand public et aux artistes »-, la chanson devait servir d'inspiration poétique : «La chanson d'autrefois, tirée de l'oubli, a beaucoup conservé de sa fraîcheur et de sa beauté ; elle peut inspirer le poète et le musicien. Le souffle qui l'anime se propage dans une ambiance vibrante, créatrice. Elle conduit à de nouvelles œuvres ». Il note son caractère populaire et éprouve l'obligation de la rendre au peuple : «Sa lyre a aussi le don de réveiller l'enthousiasme. Il y a en elle quelque chose de collectif. Elle parle à la foule ; elle en est comprise, elle qui jadis y vécut, y voyagea et s'y perdit après bien des égarements ». Mais elle était utile à chacun dans l'exercice de son travail ou dans les loisirs : « toujours humble, elle restait la compagne fidèle de la ménagère à son foyer, du laboureur dans son champ, et du canotier le long des rivières. Sans elle, le rythme du labeur se serait alangui. La vie, dans le silence, aurait été bien morne ». Dans la société contemporaine, elle a toujours sa place. 


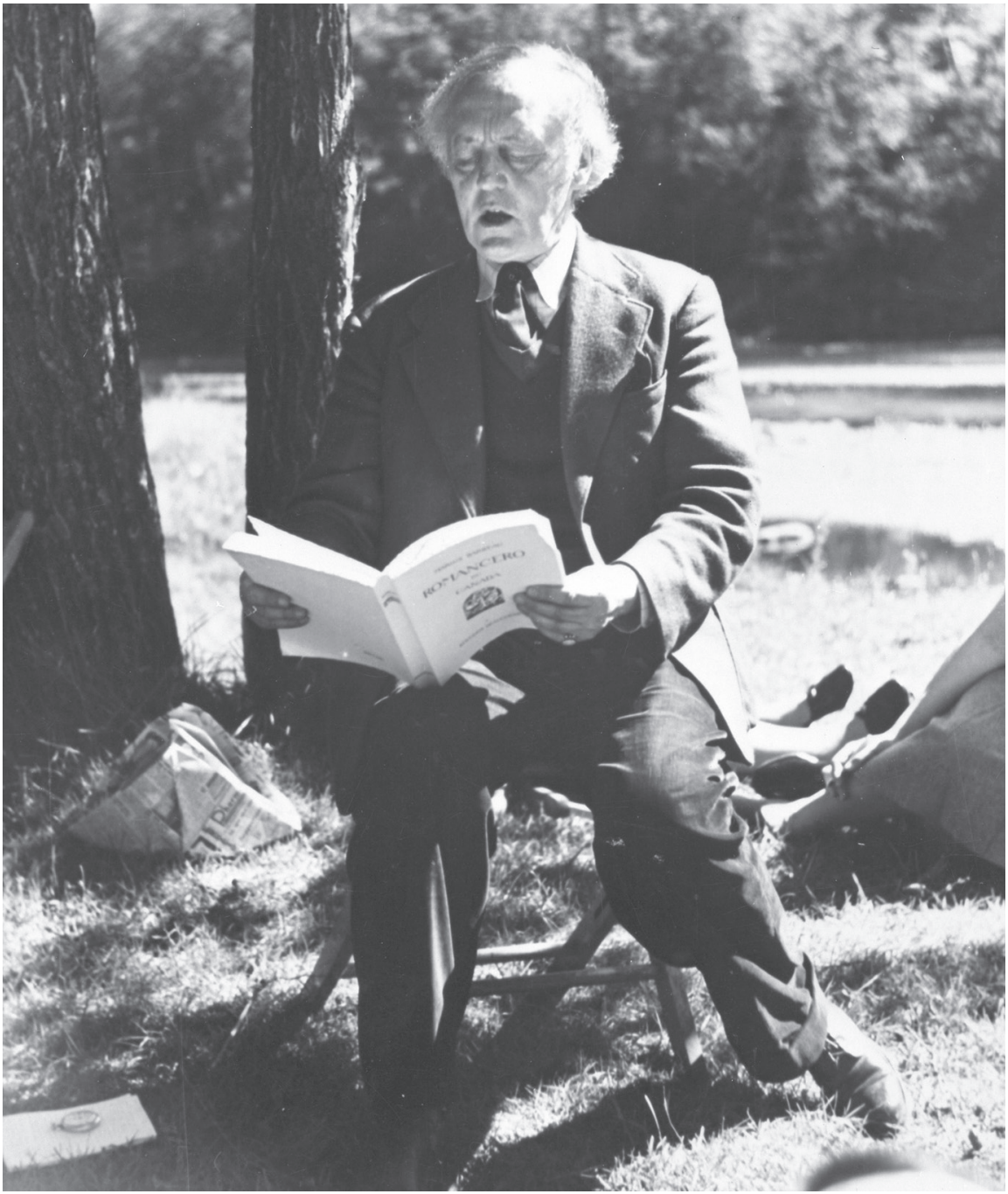

Marius Barbeau lisant un extrait de son livre Romancero du Canada à un auditoire à l'extérieur, vers 1945.

Source : Musée canadien de l'histoire, $\mathrm{n}^{\circ}$ 86-1252

\section{Un Romancero problématique}

Le Romancero du Canada eut indéniablement pour modèle Le Romancéro populaire de la France, un ouvrage posthume de George Doncieux (1856$1903)^{6}$. Barbeau était familier avec cette anthologie depuis un bon moment déjà. Elle lui avait même servi de guide dès 1919 pour la présentation

6. George Doncieux, Le Romancéro populaire de la France. Choix de chansons populaires françaises. Textes critiques par George Doncieux avec un avant-propos et un index musical par Julien Tiersot, Paris, Librairie Émile Bouillon, éditeur, 1904, xuIv-522 p. 
des quarante-six textes des Chants populaires $d u$ Canada de la collection Massicotte. Il avait trouvé dans l'établissement du texte critique un moyen commode de rendre compte des variantes d'une chanson. Il lui emprunta son titre, « romancero », le nombre de pièces traitées, les principaux éléments de son ordonnance, la reconstitution d'un texte critique, dit primitif, même la disposition particulière des vers longs pour tenir compte de la loi des césures inverses et jusqu'à l'écriture de certains mots ( $m$ 'amie au lieu de ma mie) ; en outre, Barbeau se reportera régulièrement aux commentaires de Doncieux pour les douze chansons communes aux deux ouvrages.

Si l'ethnologue multipliait ses efforts dans plusieurs directions pour faire connaître ses chansons, il ne perdit jamais de vue son projet fondamental. Et c'est avec cette préoccupation en tête qu'il se rendit en Europe au printemps de 1931 pour un séjour de trois mois. Il s'était donné pour mission « d'inventorier les collections indiennes dans les musées de Paris et d'Angleterre », mais aussi « de poursuivre en même temps une étude comparée sur les chansons folkloriques canadiennes-françaises ». Il était devenu important, pour l'achèvement de son recueil, d'obtenir des données comparatives, «notamment à la Bibliothèque nationale où [il] désirai[ $\mathrm{t}$ ] consulter ce qu['il] croyai[t] être l'unique exemplaire de la collection Ampère »; en somme, confiera-t-il dans ses « Mémoires » : « Je voulais savoir, par exemple, quelles chansons canadiennes-françaises avaient déjà été signalées en France ${ }^{7} »$. Pendant son séjour à Paris, il fera la rencontre de Raoul et Marguerite d'Harcourt, dont il connaissait la réputation d'ethnomusicologues, et qui fut décisive pour la publication de son Romancero du Canada. Un grand projet de collaboration naquit d'ailleurs de cette relation : la transcription d'un millier de chansons du répertoire Barbeau qui mènera à plusieurs publications, culminant dans une étude musicale de deux cent quarante versions des Chansons folkloriques françaises au Canada en 1956. Mais, assez rapidement, $\mathrm{M}^{\mathrm{me}}$ Béclard d'Harcourt acceptera d'abord de faire l'analyse musicale des chansons du Romancero, puisque son nom figure déjà dans l'avant-propos du recueil daté du 31 mai 1932, et elle rédigera enfin la préface en juin 1936.

Ainsi, il appert que le projet, annoncé dès 1919 dans les Chants populaires du Canada (Première série) et mis peu après en veilleuse, n'avait cessé de poursuivre Marius Barbeau comme on le voit encore par les publications qu'il consacra à la chanson populaire et dont la plupart contenaient une ou plusieurs des pièces qui seront réunies dans l'anthologie de 1937. Ces nombreuses rééditions n'apaisèrent vraisemblablement pas sa soif de diffusion, car il consacra une partie de sa retraite à son projet initial, remodelé en « répertoire de la chanson folklorique française au Canada » qu'il entendait distribuer en

7. «Les Mémoires de Marius Barbeau », enregistrés et transcrits par Carmen Roy, Ottawa, Musée canadien de l'histoire, 1958, p. 211. 
quatre volumes : « Le rossignol y chante », « En roulant ma boule », « Le roi boit » et «Envoyons d'l'avant, nos gens ! » Au moment de sa mort, en 1969, il avait déjà rédigé trois recueils et publié le premier d'entre eux ; les deux recueils posthumes furent préparés par Lucien Ouellet, du Musée canadien des civilisations. Parmi les 656 chansons du répertoire, toutes les pièces du Romancero du Canada y trouveront leur place.

\section{Conclusion}

La grande originalité de l'œuvre de Marius Barbeau, tant pour la chanson que pour le conte, réside dans la quête inédite de la tradition orale qu'il enclencha en 1914 et qu'il surprit, dans sa vivante authenticité, chez des témoins inattendus de régions périphériques : le pays de Charlevoix, la Beauce et le Bas-Saint-Laurent. En scientifique véritable et sans idée préconçue, à l'opposé d'Ernest Gagnon dont le recueil remarquable s'inscrivait dans un débat autour du chant grégorien, il suivait son instinct de collectionneur, tout enchanté de constater que ce devancier n'avait pas tout publié. Chercheur curieux, il s'émerveilla des moissons qu'il engrangeait sans cesse dans ses archives et dont l'inventaire le troublait. Il s'étonna encore davantage quand l'analyse lui révéla la grande qualité des chansons recueillies dont l'état de conservation faisait l'envie de la mère-patrie. Et il voulut les faire connaître au public. Ce goût inaltérable de publier ces refrains d'autrefois, sous toutes les formes possibles, dans des journaux et des magazines, dans des revues et des livres, par des concerts et des créations artistiques, tenait de sentiments ambivalents : d'une part, l'impression d'être l'ultime témoin d'une tradition moribonde : « Mais aujourd'hui, le souffle s'est affaibli, et la tradition poétique n'a pas même survécu parmi le peuple. C'en est fini de la chanson »; d'autre part, le besoin de rendre au peuple, sous des formes naturelles ou adaptées, la grande tradition qu'il avait canalisée et qu'il désirait relancer. Ainsi, Barbeau, qui l'a favorisée en son temps, apprécierait sans doute la réinterprétation artistique contemporaine de la chanson traditionnelle et les vagues périodiques qui la renouvellent depuis 1970 selon les modes du jour.

La publication des paroles et de la musique, modèle qu'Ernest Gagnon avait inauguré au Canada, n'allait pas de soi à l'époque. Marius Barbeau y adhérait pourtant, convaincu qu'il fallait «s'intéresser réellement aux chansons de folklore et savoir les transcrire avec la musique, afin de placer les mélodies et les paroles ensemble, de façon à les rendre propres à la publication ». Puis il trouva chez Doncieux un modèle propre, croyait-il, à rendre compte, avec une économie de moyens, de la pluralité de la tradition.

Intuitif, Barbeau l'était certainement. Il le reconnut un jour en confiant ses souvenirs à Carmen Roy : « Maintenant, je vais vous avouer - et il s'agit là peut-être d'un secret dans ma vie - ce besoin que j'ai toujours éprouvé 
d'avancer dans une direction qui débouche sur quelque chose d'intéressant et d'en savoir saisir l'attrait. C'est en quelque sorte la force motrice de ma vie, je crois, d'aller vers quelque chose que je sais là, quelque chose d'intéressant qui n'a pas encore été atteint ${ }^{8} \gg$. Les intuitions dont Barbeau faisait alors état ont été à l'origine de sa féconde et multiple carrière, comme elles ont été à la source aussi de la fondation de la chaire des Archives de folklore et du programme d'enseignement qu'elle engendra à l'Université Laval, puis de l'orientation disciplinaire des Luc Lacourcière, Germain Lemieux, JosephThomas LeBlanc, Anselme Chiasson et Conrad Laforte, sans compter les destins artistiques suscités au cours des générations qui ont suivi l'année $1937^{9}$.

8. Ibid., p. 49.

9. Pour une étude plus complète de cet ouvrage, voir Jean-Pierre Pichette, « Le Chercheur de trésors ou l'influence d'un livre. Marius Barbeau et le Romancero du Canada », dans les Cahiers Charlevoix 7. Études franco-ontariennes, Sudbury, Société Charlevoix et Prise de parole, 2006 [2007], p. $85-141$.

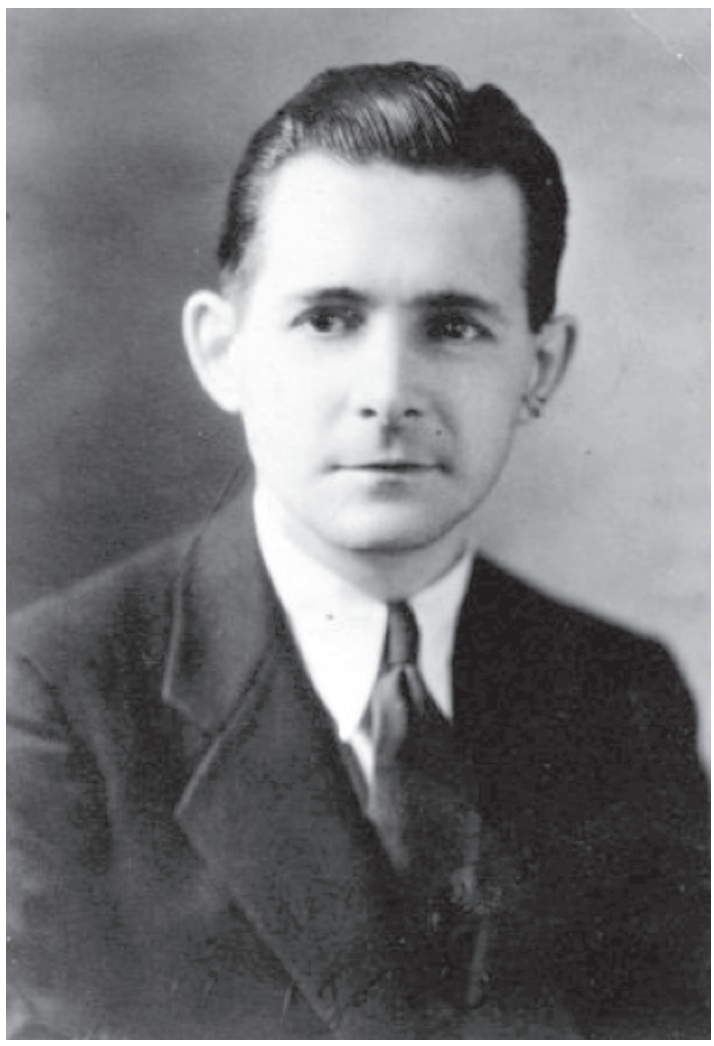

Joseph-Thomas LeBlanc (s.d)

Source : AfEul P178-C5-2. 